ARTICLE

DOI: $10.1038 / \mathrm{s} 41467-018-03656-4$

\title{
Ultrastable metallic glasses formed on cold substrates
}

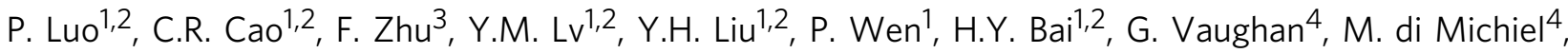 \\ B. Ruta (iD ${ }^{4,5} \&$ W.H. Wang 1,2
}

Vitrification from physical vapor deposition is known to be an efficient way for tuning the kinetic and thermodynamic stability of glasses and significantly improve their properties. There is a general consensus that preparing stable glasses requires the use of high substrate temperatures close to the glass transition one, $T_{g}$. Here, we challenge this empirical rule by showing the formation of Zr-based ultrastable metallic glasses (MGs) at room temperature, i.e., with a substrate temperature of only $0.43 T_{\mathrm{g}}$. By carefully controlling the deposition rate, we can improve the stability of the obtained glasses to higher values. In contrast to conventional quenched glasses, the ultrastable MGs exhibit a large increase of $T_{\mathrm{g}}$ of $\sim 60 \mathrm{~K}$, stronger resistance against crystallization, and more homogeneous structure with less order at longer distances. Our study circumvents the limitation of substrate temperature for developing ultrastable glasses, and provides deeper insight into glasses stability and their surface dynamics.

\footnotetext{
${ }^{1}$ Institute of Physics, Chinese Academy of Sciences, 100190 Beijing, China. ${ }^{2}$ University of Chinese Academy of Sciences, 100049 Beijing, China. ${ }^{3}$ State Key Laboratory of Metal Matrix Composites, School of Materials Science and Engineering, Shanghai Jiao Tong University, 200030 Shanghai, China. ${ }^{4}$ ESRF-The European Synchrotron, CS40220, 38043 Grenoble, France. ${ }^{5}$ Univ Lyon, Université Claude Bernard Lyon 1, CNRS, Institut Lumière Matière, 69622

Villeurbanne, France. Correspondence and requests for materials should be addressed to W.H.W. (email: whw@iphy.ac.cn)
} 
G lasses are generally produced by cooling a liquid very rapidly to circumvent crystallization ${ }^{1,2}$. The glass transition is a kinetic process ${ }^{1,2}$, and the slower is the cooling rate, the larger is the time available for the supercooled liquid to rearrange its structure and follow the temperature changes before to freeze in the glassy state. Following this observation, one could think to tune the properties of a glass by simply controlling the rate of cooling. However, the effect of the cooling rate is quite small, as one order of magnitude faster rate merely increases the glass transition temperature, $T_{\mathrm{g}}$, by $\approx 3 \mathrm{~K}$ in both polymers ${ }^{3}$ and metallic glasses (MGs) ${ }^{4}$. This increment is definitely too small for the majority of technological purposes.

In the past decade, Ediger and co-workers demonstrated that by virtue of physical vapor deposition the kinetic limitations in traditional glass formations can be bypassed, and it is possible to synthesize organic molecular glasses with extraordinary thermodynamic and kinetic stability ${ }^{5}$, and exceptional mechanical properties ${ }^{6}$. These glasses, known as ultrastable glasses, exhibit much higher $T_{\mathrm{g}}$ with respect to their ordinary counterparts produced by conventional liquid cooling. Similar properties would be expected from liquid-cooled glasses only after aging for thousands of years or more ${ }^{7,8}$. In subsequent efforts, more species of ultrastable glasses, such as MGs ${ }^{9,10}$ were fabricated by vapor deposition, and it has been possible to obtain even Lennard-Jones ultrastable glasses ${ }^{11}$ by numerical simulations. In all these works, the general consensus is that the substrate temperature $\left(T_{\text {sub }}\right)$ during deposition is a critical variable, and that the optimal $T_{\text {sub }}$ for creating ultrastable glasses is near the $T_{\mathrm{g}}$ of ordinary glasses, e.g., $T_{\text {sub }}=0.8 \sim 0.9 T_{\text {g }}$ (refs. ${ }^{5-12}$ ).

It has been demonstrated in various glasses that the surface mobility is several orders of magnitude faster and exhibits weaker temperature dependence than that of the bulk ${ }^{13-18}$. This enhanced surface mobility is thought to play a key role in the formation of ultrastable glasses via vapor deposition ${ }^{5-12}$. By increasing $T_{\text {sub }}$, the mobility of atoms or molecules at the surface is significantly enhanced. This allows the constituents to sufficiently reorganize and explore stable configurations, resulting then in the formation of ultrastable glasses ${ }^{5-12}$. Although the creation of ultrastable polymer glasses by the assembly of polymer nanoglobules is different from typical vapor deposition, a high $T_{\text {sub }} \approx 0.87 T_{\mathrm{g}}$ is still required ${ }^{19}$. The coincidence of the optimal $T_{\text {sub }}$ for preparing ultrastable glasses with the ideal glass transition temperature (Kauzmann temperature) has also led to the argument that there is an underlying thermodynamic mechanism governing the formation of ultrastable glasses ${ }^{11}$. Following all these works ${ }^{5-12,19}$, high $T_{\text {sub }}$ near $T_{\mathrm{g}}$ has been considered as a prerequisite in synthesizing ultrastable glasses, while low $T_{\text {sub }}$ has been suggested to decrease both the thermodynamic role and the surface dynamics ${ }^{12}$.

Here, we contradict this empirical rule by showing that it is possible to obtain ultrastable MGs on cold substrate with $T_{\text {sub }}$ far below $T_{\mathrm{g}} \quad\left(T_{\text {sub }} \approx 0.43 T_{\mathrm{g}}\right)$. Substantial enhancement of glass stability can be achieved by lowering the deposition rate, $R$ to $\sim 1 \mathrm{~nm} \mathrm{~min}{ }^{-1}$. In comparison with ordinary glasses obtained by liquid quenching, our glasses exhibit much higher stability with an increase of $T_{\mathrm{g}}$ up to $\sim 60 \mathrm{~K}$, a more homogeneous structure, and a stronger resistance to crystallization. More importantly, our work suggests that the relaxation dynamics at the surface of the glass could be much faster-than-expected despite of the low temperature substrate, and call for a reconsideration of the prerequisites for ultrastability of glasses.

\section{Results}

Experiment. A $\mathrm{Zr}_{46} \mathrm{Cu}_{46} \mathrm{Al}_{8}$ (at.\%) target was utilized for ion beam assisted deposition (IBAD). Different from previous works, a

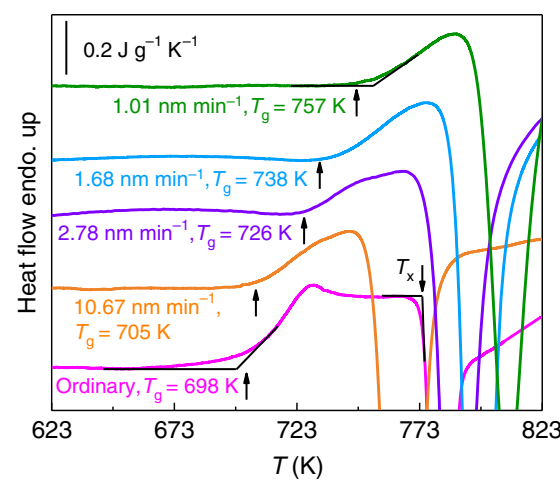

b

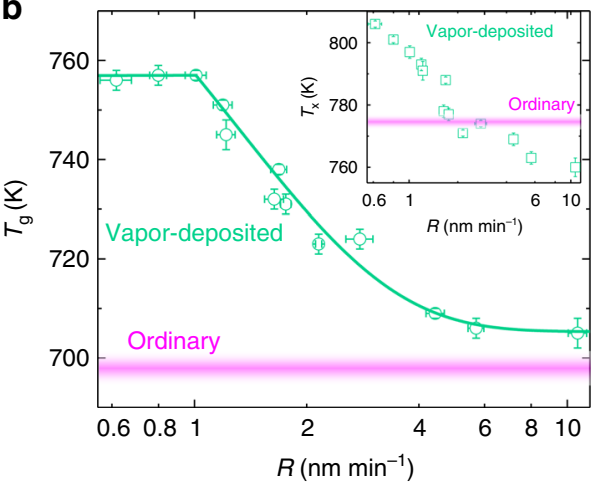

Fig. 1 Effect of vitrification routes on the glass transition and crystallization. a Representative DSC traces at a heating rate of $20 \mathrm{~K} \mathrm{~min}^{-1}$ for

$\mathrm{Zr}_{46} \mathrm{Cu}_{46} \mathrm{Al}_{8} \mathrm{MGs}$ : ordinary glass produced by melt-spinning technic; vapor-deposited glass films at different rates as denoted below each curve. $T_{\mathrm{g}}$ and $T_{\mathrm{x}}$ are defined from the onset of the transformation as indicated by the intersection of the black lines. $\mathbf{b} T_{\mathrm{g}} \mathrm{vs}$. $R$. The solid line at high $R$ is an exponential fit to the data, at low $R$ the $T_{\mathrm{g}}$ keeps invariant. Inset: $T_{\mathrm{x}} \mathrm{vs}$. $R$. The error bars indicate the standard deviation of three to five

measurements. For comparison, the $T_{\mathrm{g}}$ and $T_{\mathrm{x}}$ with their variation ranges for the ordinary glass are presented by the shaded magenta areas

we leave the substrate at room temperature which corresponds to $T_{\text {sub }} \approx 0.43 T_{\mathrm{g}}$. Details about the sample preparation and subsequent measurements can be found in the Methods section. Chemical analysis (Thermo IRIS Intrepid II XSP) confirmed that the compositions of the vapor-deposited glass films are identical to that of the ordinary glass ribbon prepared by melt-spinning method within $1 \%$ error (Supplementary Table 1). This is further ascertained by their similar melting temperatures (Supplementary Figure 1). No detectable oxygen in the deposited films was found, as confirmed by an oxygen and nitrogen analyzer. The deposited MG films have atomic level smooth and homogeneous surface structure with a root-mean-square surface roughness $\approx 0.1 \mathrm{~nm}$ (Supplementary Figure 2).

Calorimetric analyses. Figure 1 a shows the differential scanning calorimetry (DSC) profiles of $\mathrm{Zr}_{46} \mathrm{Cu}_{46} \mathrm{Al}_{8}$ MG obtained with different preparation routes. The magenta curve is for the ordinary glass prepared by liquid quenching, and its $T_{\mathrm{g}}$ is $698 \mathrm{~K}$ consistent with previous reports ${ }^{20}$. The other DSC curves are for glass films synthesized by vapor deposition at different rates. As can be seen, $T_{\mathrm{g}}$ increases with decreasing deposition rate. When the deposition rate is $10.67 \mathrm{~nm} \mathrm{~min}^{-1}$, the glass film has a similar $T_{\mathrm{g}}(705 \mathrm{~K})$ to that of the ribbon. As the deposition rate is lowered to $1.01 \mathrm{~nm} \mathrm{~min}{ }^{-1}$, the $T_{\mathrm{g}}$ of the corresponding glass increases to $757 \mathrm{~K}$, which is $59 \mathrm{~K}$ higher than that of the ordinary glass. This $8.5 \%$ increase of $T_{\mathrm{g}}$ at $T_{\text {sub }} \approx 0.43 T_{\mathrm{g}}$ is even larger than the 

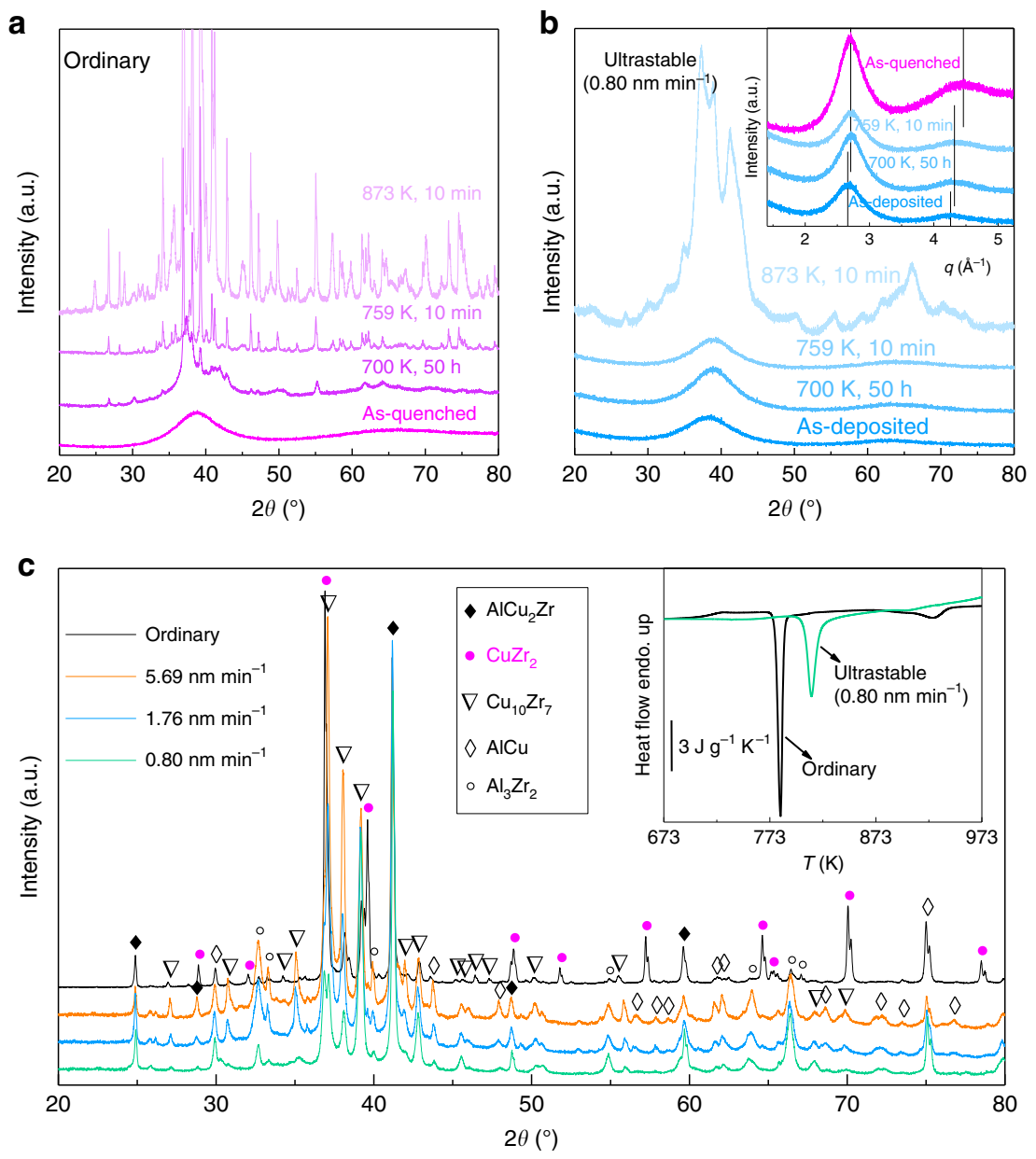

Fig. 2 Crystallization dynamics and phase formation of the ordinary and the ultrastable glasses upon annealing. $\mathbf{a}$, $\mathbf{b}$ XRD profiles for as-prepared and annealed $\mathbf{a}$ ordinary and $\mathbf{b} 0.80 \mathrm{~nm} \mathrm{m^{-1 }}$ deposited ultrastable MGs. The annealing temperature and time are denoted by the legend values above each curve. Inset in $\mathbf{b}$ : XRD data for as-deposited and annealed ultrastable MGs and as-quenched ordinary MGs plotted as a function of $q=4 \pi \sin \theta / \lambda$.

The vertical lines indicate the peak positions. c XRD profiles for samples after annealed at $973 \mathrm{~K}$ for $1 \mathrm{~h}$, from top to bottom, the ordinary, $5.69 \mathrm{~nm}$ min ${ }^{-1}$, $1.76 \mathrm{~nm} \mathrm{~min}^{-1}$, and $0.80 \mathrm{~nm} \mathrm{~min}{ }^{-1}$ vapor-deposited glasses. Inset: DSC traces for the ordinary and the $0.80 \mathrm{~nm} \mathrm{~min}^{-1}$ deposited glasses at a heating rate of $20 \mathrm{Kmin}^{-1}$

previously reported value $(7.1 \%)$ at high $T_{\text {sub }} \approx 0.8 T_{\mathrm{g}}$ for a $\mathrm{Zr}_{55} \mathrm{Cu}_{30} \mathrm{Ni}_{5} \mathrm{Al}_{10} \mathrm{MG}$ (ref. ${ }^{10}$ ). At the same $T_{\text {sub }}$ of $0.8 T_{\mathrm{g}}$, Yu et al. reported instead an increase of $T_{\mathrm{g}}$ by only $1.6 \%$ for a $\mathrm{Zr}_{65} \mathrm{Cu}_{27.5} \mathrm{Al}_{7.5} \mathrm{MG}^{9}$. This difference with the pioneering work of $\mathrm{Yu}$ et al. ${ }^{9}$ is likely due to the employed deposition rate of $84 \mathrm{~nm} \mathrm{~min}^{-1}$, much higher than that used in ref. 10 $\left(11.4 \mathrm{~nm} \mathrm{~min}^{-1}\right)$ and in our study. These comparisons highlight thus the importance of the deposition rate in improving the stability of the deposited glass.

Figure $1 \mathrm{~b}$ shows the semi-log plot of $T_{\mathrm{g}}$ vs. $R$ for the vapordeposited glasses, together with the $T_{\mathrm{g}}$ of the ordinary ribbon (shaded magenta area). For $R<1.01 \mathrm{~nm} \mathrm{~min}^{-1}, T_{\mathrm{g}}$ appears constant around $757 \mathrm{~K}$. This plateau at low $R$ sets a lower limit on the experimentally meaningful deposition rate, as further decreasing $R$ does not lead to higher $T_{\mathrm{g}}$ within the experimental sensitivity. When $R$ is larger than $1.01 \mathrm{~nm} \mathrm{~min}^{-1}, T_{\mathrm{g}}$ decreases exponentially with increasing $R$, and at $R \approx 10 \mathrm{~nm} \mathrm{~min}^{-1}, T_{\mathrm{g}}$ gradually approaches the value of the ribbon. It is clear that the kinetic stability as quantified by the value of $T_{\mathrm{g}}$ is strongly influenced by the rate of deposition: lowering the deposition rate by merely one order of magnitude leads to significantly enhanced $T_{\mathrm{g}}$ by as large as $60 \mathrm{~K}$. Interestingly, $T_{\mathrm{x}}$ is also affected by the deposition rate. This is shown in the inset in Fig. $1 b$ where the onset temperature of crystallization, $T_{\mathrm{x}}$, vs. $R$ is presented. Different from the non-monotonic variation of $T_{\mathrm{g}}, T_{\mathrm{x}}$ increases monotonically with decreasing the deposition rate. For $R>2 \mathrm{~nm} \mathrm{~min}^{-1}$, the $T_{\mathrm{x}}$ of the vapor-deposited glasses is even lower than that of the ordinary glass. For lower $R$, the increased $T_{\mathrm{x}}$ suggests the higher stability against crystallization of the ultrastable glasses.

Surface mobility. The formation of ultrastable MGs at $T_{\text {sub }}$ far below $T_{\mathrm{g}}$ suggests that the atoms at the surface still retain sufficient mobility even at lower temperatures. The slow rate of deposition makes the atoms have enough time to rearrange on the surface, and the system can explore more stable configurations before buried by the upcoming atoms. When the deposition rate is lower than $1.01 \mathrm{~nm} \mathrm{~min}^{-1}$, the value of $T_{\mathrm{g}}$ reaches a plateau, suggesting that the timescale set by this critical rate is close to that of the surface dynamics. The free surface residence time ${ }^{21}$ before a fresh atomic layer is deposited can be estimated by dividing the thickness of one monolayer by the deposition rate. The atomic weighted diameter, as calculated by weighting the atomic diameter of each component to their composition, can be taken as a measure of the thickness of the top monolayer. Given the atomic radius of $0.160 \mathrm{~nm}$ for $\mathrm{Zr}, 0.128 \mathrm{~nm}$ for $\mathrm{Cu}$, and $0.143 \mathrm{~nm}$ for $\mathrm{Al}$, the thickness of the surface monolayer is estimated to be 
$\approx 0.288 \mathrm{~nm}$. At the deposition rate of $1.01 \mathrm{~nm} \mathrm{~min}^{-1}$, the free surface residence time is therefore estimated to be $\approx 17 \mathrm{~s}$. Based on the fact that further lowering the deposition rate will not affect the $T_{\mathrm{g}}$ value (Fig. 1b), it is reasonable to conclude that $17 \mathrm{~s}$ is long enough for atoms at the free surface to rest and freely explore stable configurations before buried. Successive layer by layer deposition results thus in a glass with highly enhanced stability. Following this argument, the timescale associated with the surface dynamics should be close to $17 \mathrm{~s}$ as well.

Crystallization upon annealing. The significantly improved stability of our ultrastable glasses is further confirmed by studies on the kinetics of the structural evolution under isothermal annealing. By means of laboratory X-ray diffraction (XRD), we characterized the structure of both ordinary and ultrastable MGs heated at $20 \mathrm{~K} \mathrm{~min}^{-1}$ to different annealing temperatures. Figure $2 \mathrm{a}, \mathrm{b}$ shows, respectively, the XRD patterns for the ordinary and the ultrastable MGs, in the as-prepared and annealed states. The presence of a unique broad diffraction peak in the as-prepared samples indicate their fully amorphous nature. After annealing at $700 \mathrm{~K}$ for $50 \mathrm{~h}$, and at $759 \mathrm{~K}$ and $873 \mathrm{~K}$ for 10 min, sharp Bragg peaks emerge in the XRD spectra of the ordinary glass, indicating the precipitation of crystalline phases (Fig. 2a). In contrast, the ultrastable glasses are more resistant against crystallization (Fig. 2b). They remain still completely amorphous after annealing at $700 \mathrm{~K}$ and $759 \mathrm{~K}$. To trigger the crystallization, it is necessary to anneal the glass at much higher temperature $(873 \mathrm{~K})$. In this case, DSC measurements indicate that crystallization in the ultrastable glasses has been completed (inset in Fig. 2c). In contrast to the ordinary glass, no well-defined Bragg peaks show up in the ultrastable glasses during this annealing, suggesting that the precipitated phases are smaller in size and not well ordered in structure. These observations demonstrate the distinctly different crystallization process in our ultrastable glasses. While crystallization in the ordinary glass occurs through fast nucleation and grain growth, our results suggest that there are probably more nucleation sites in the ultrastable glasses, but the grain growth is very slow. This hypothesis is supported by DSC measurements (inset in Fig. 2c). The crystallization process in the ordinary glass covers a range of $\approx 14 \mathrm{~K}$ with a sharp exothermal peak, and it takes only $\approx 0.7 \mathrm{~min}$ to complete at the heating rate of $20 \mathrm{~K} \mathrm{~min}^{-1}$. For the ultrastable glass, the crystallization initiates, however, at much higher temperature and covers a broader range of $\approx 27 \mathrm{~K}$. These results confirm the high kinetic stability of the glasses formed at slow deposition rate, in agreement with their enhanced $T_{\mathrm{g}}$.

Furthermore, the precipitated crystalline phases in the ultrastable glasses are found to be different from that in the ordinary glass, strengthening the idea of the presence of different local atomic configurations. To compare the phase formation, the glasses were annealed at $973 \mathrm{~K}$ for $1 \mathrm{~h}$. The annealing temperature is below the onset temperature of the B2 phase transition in this alloy ${ }^{22}$, so that the possible effect of crystalline phase transition can be excluded. Figure $2 c$ displays the XRD patterns for the annealed ordinary and vapor-deposited glasses. In the annealed ribbon, the precipitated phases are identified to be mainly intermetallic $\mathrm{AlCu}_{2} \mathrm{Zr}$ and $\mathrm{CuZr}_{2}$ with minor amount of $\mathrm{AlCu}, \mathrm{Cu}_{10} \mathrm{Zr}_{7}$, and $\mathrm{Al}_{3} \mathrm{Zr}_{2}$. Differently, the XRD patterns for the annealed ultrastable glass does not show the presence of $\mathrm{CuZr}_{2}$ phase, and the primary phases in the ultrastable glasses are $\mathrm{AlCu}_{2} \mathrm{Zr}$ and $\mathrm{Cu}_{10} \mathrm{Zr}_{7}$. This indicates that the precipitation of $\mathrm{CuZr}_{2}$ is suppressed in the ultrastable glasses. The patterns for the vapor-deposited glasses are very similar, but the peak intensity of $\mathrm{Cu}_{10} \mathrm{Zr}_{7}, \mathrm{AlCu}$, and $\mathrm{Al}_{3} \mathrm{Zr}_{2}$ phases increases as the deposition rate is increased, indicating
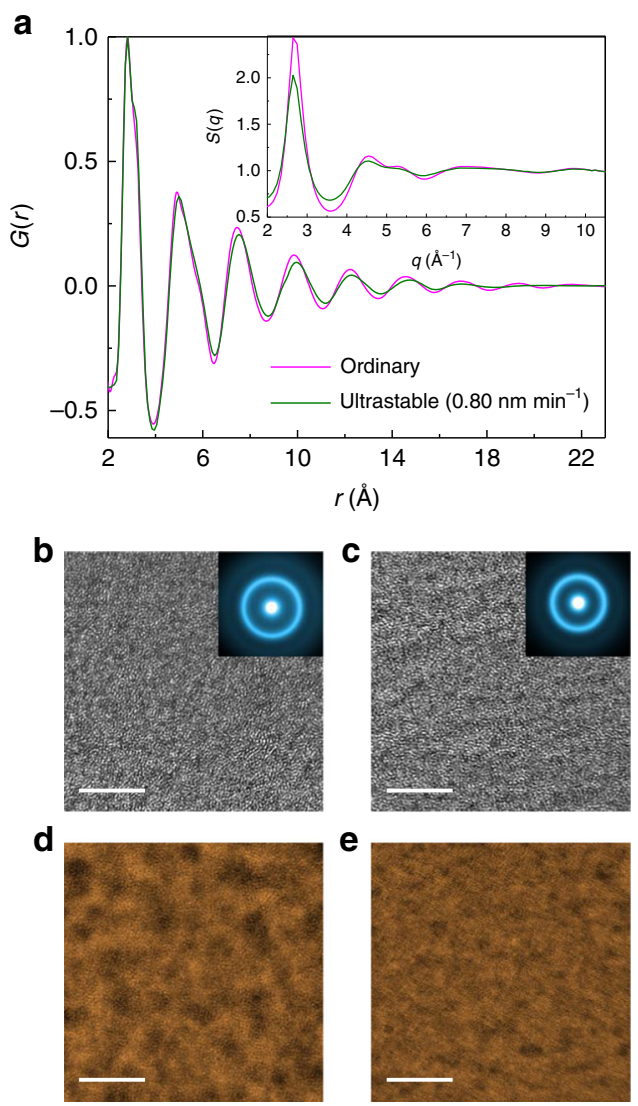

Fig. 3 Structure characterization for the ordinary and the $0.80 \mathrm{~nm} \mathrm{~min}^{-1}$ deposited ultrastable glasses. a Pair distribution function $G(r)$ and static structure factor $S(q)$ (the inset) for the ordinary and the ultrastable MGs. b, c HRTEM images of $\mathbf{b}$ the ordinary and $\mathbf{c}$ the ultrastable MGs. Insets: selected area electron diffraction (SAED) patterns taken from a large selected area. $\mathbf{d}$, e HADDF-STEM images of $\mathbf{d}$ the ordinary and $\mathbf{e}$ the ultrastable MGs. The scale bars in $\mathbf{b}$-e are $5 \mathrm{~nm}$

the effect of deposition rate on the formation of crystalline phases.

Structure characterization. The different crystallization dynamics and phase precipitation between the ultrastable and the ordinary glass indicate that substantial structural changes have been imparted to the glass during the deposition. Indeed, a closer look at Fig. 2a, b shows that the two as-prepared glasses do not have the same structure. The inset in Fig. $2 b$ shows the XRD data for the as-deposited and annealed ultrastable MGs and the asquenched ordinary MGs as a function of the wave vector $q$, being $q=4 \pi \sin \theta / \lambda$, and $\theta$ and $\lambda$ the scattering angle and the wavelength of the incident beam, respectively. With respect to the ordinary glass, the ultrastable glass shows less intense and broader peaks, slightly shifted toward lower $q$ values. These structural differences are confirmed also by high-energy synchrotron XRD measurements (see the static structure factor $S(q)$ in the inset in Fig. 3a) and correspond to changes in the local atomic structure. These changes can be better appreciated by real space analysis, through the evaluation of the pair distribution functions, $G(r)$. As shown in Fig. 3a, the $G(r)$ of the ordinary glass presents well-defined oscillations up to the 8th neighboring shell, while the ultrastable glass is more glassy and exhibits an ordered structure only up to the 6 th shell. This means that the ordinary glass is more ordered at longer distances, and is characterized by more packed shells, as 


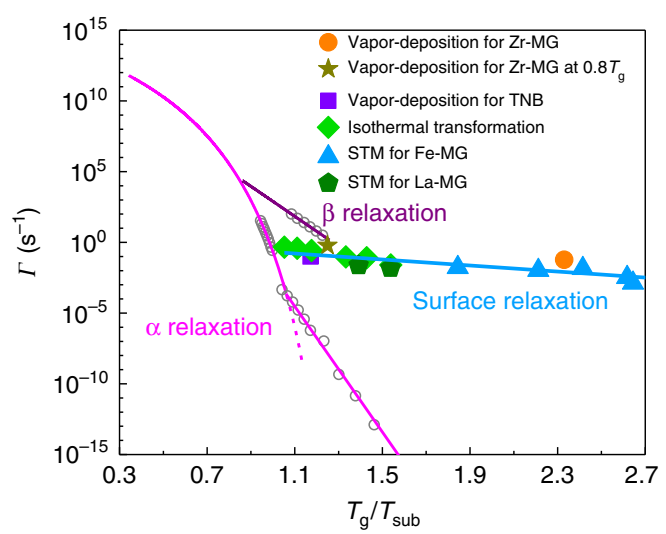

Fig. 4 Comparison of surface relaxation with bulk $\alpha$ and $\beta$ relaxations. The magenta and purple curves along with the open circles show the bulk $\alpha$ and $\beta$ relaxation rates vs. $T_{g} / T$ in $M_{G s}{ }^{35}$, respectively. The solid square, star, and circle show the surface relaxation rate $\Gamma_{\text {surface }}$ vs. $T_{g} / T_{\text {sub }}$ estimated from vapor deposition of an ultrastable molecular glass ${ }^{5}$, the $\mathrm{Zr}-\mathrm{MG}$ of ref. ${ }^{10}$, and the sample studied in the present work, respectively. The rhombuses are for ethylcyclohexane glass as $\Gamma_{\text {surface }}$ is the inverse critical free surface residence time at which the time of isothermal transformation from the glass into the supercooled liquid state reaches the plateau (Figure 9 in ref. ${ }^{21}$ ). The $\Gamma_{\text {surface }}$ from STM measurements are plotted as pentagons (left one: $\mathrm{La}_{50} \mathrm{Ni}_{15} \mathrm{Al}_{25} \mathrm{Cu}_{10}$, right one: $\mathrm{La}_{60} \mathrm{Ni}_{15} \mathrm{Al}_{25} \mathrm{MGs}^{32}$ ) and as triangles $\left(\mathrm{Fe}_{78} \mathrm{~B}_{13} \mathrm{Si}_{9} \mathrm{MG}^{33},{ }^{34}\right)$. The blue line is a linear fit to the surface relaxation rates

suggested by the shifts of the peaks of the $G(r)$ toward shorter distances. Interestingly, structural modifications have been reported also for organic glasses produced by vapor deposition at high $T_{\text {sub }}$ (refs. ${ }^{23,27}$ ).

Further structural characterizations have been performed also by using transmission electron microscopy (TEM). The highresolution TEM (HRTEM) images appear to be similar for both ordinary (Fig. 3b) and ultrastable glass (Fig. 3c), such as the homogeneous maze-like patterns. Differently, scanning transmission electron microscopy (STEM) is more sensitive to the local structure and can project the local atomic arrangements in real space. Large variations in contrast can be observed in the ordinary glass by high-angle annular dark-field scanning TEM (HADDF-STEM) (Fig. 3d), indicating its heterogeneous atomic arrangements $^{28}$. On the contrary, the contrast variation is much lower in the ultrastable glass (Fig. 3e), indicative of a more homogeneous atomic structure. Since all TEM specimens are prepared by ion milling under the same conditions, such difference between the ultrastable and the ordinary glass can be attributed to their distinct structural packing and configuration, in agreement with the XRD results. In molecular systems, the enhanced stability can be correlated with molecular orientation or layered packings ${ }^{25,26,29}$. Differently, MGs are made of isotropic constituents, ruling out the possible effect from molecular orientation. However, as a multi-component material, further investigations are warranted to distinguish the role of the local atomic structure and layered packing in the increased stability of MG, which is crucial for clarifying the process of producing stable MG.

\section{Discussion}

The unique deposition rate dependence of $T_{\mathrm{g}}$ shown in Fig. $1 \mathrm{~b}$ sheds additional light on the surface properties of MGs, especially at such a low $T_{\text {sub. The increasing stability with decreasing }}$ deposition rate supports the idea of an enhanced surface mobility mechanism, analogous to the one occurring at high $T_{\text {sub }}^{7,21}$. In addition, the fact that just one order of magnitude lower rate of deposition generates a huge enhancement in glass stability even at a $T_{\text {sub }}$ far below the Kauzmann temperature ${ }^{30}$ (Fig. 1b), questions the argument of an underlying thermodynamic mechanism governing the formation of stable glasses ${ }^{11}$. In numerical simulations $^{31}$, it was found that the optimal $T_{\text {sub }}$ for a given deposition rate decreases as deposition slows, suggesting a competition between thermodynamics and kinetics. This study shows that the stability keeps increasing monotonically even after four orders of magnitude decrease of the deposition rate ${ }^{31}$. In contrast, experiments on an ethylcyclohexane glass prepared at $T_{\text {sub }}=0.6 \sim 0.95 T_{\mathrm{g}}$ (ref. ${ }^{21}$ ) and our MGs obtained at $T_{\text {sub }}=0.43 T_{\mathrm{g}}$ show the occurrence of a lower limit in the increasing of the glass stability within just one to two orders of magnitude slower deposition rates. These discrepancies are likely to be a consequence of the difference in procedures for vapor deposition employed in numerical simulations and experiments, and highlight the importance of kinetics during stable glass formation with much faster-than-expected surface dynamics. From the preparation of ultrastable molecular glasses, Ediger et al. estimated a relaxation time of $10 \mathrm{~s}$ for the top surface layer at $T_{\text {sub }}=0.85 T_{\mathrm{g}}\left(\right.$ ref. $\left.^{5}\right)$. In our work, the deposition rate has been lowered to the critical limit of $\approx 1 \mathrm{~nm} \mathrm{~min}^{-1}$ below which $T_{\mathrm{g}}$ remains unaffected. The estimated surface relaxation time of $\approx 17$ $s$ is in good agreement with the direct probing of atomic rearrangements at the surface of MGs obtained by scanning tunneling microscope (STM) measurements ${ }^{32-34}$. This is shown in Fig. 4 where we report an Arrhenius diagram displaying the surface relaxation rates ( $\Gamma_{\text {surface }}$, inverse relaxation time) as a function of $T_{\mathrm{g}} / T_{\text {sub }}$ for different glasses and measured by different approaches. The data for bulk $\alpha$ and $\beta$ relaxations of MGs measured in our previous study ${ }^{35}$ are shown for comparisons, where the bulk a relaxation follows a Vogel-Fulcher-Tammann (VFT) fashion in supercooled liquid state and an Arrhenius fashion in glassy state. Despite of the diverse nature of the systems and experimental techniques used for estimating the surface relaxation rates, the data collapse on a single curve which can be described by an Arrhenius process with an activation energy of $2.4( \pm 0.4) k_{\mathrm{B}} T_{\mathrm{g}}$. This value is much smaller than that reported for the bulk $a$ $\left(\approx 52 k_{\mathrm{B}} T_{\mathrm{g}}\right)$ and $\beta\left(\approx 26 k_{\mathrm{B}} T_{\mathrm{g}}\right)$ relaxations of different $\mathrm{MGs} \mathrm{s}^{35}$. As a further confirmation of our estimation, we have also calculated the surface dynamics for the $\mathrm{Zr}$-based glass film reported in ref. ${ }^{10}$ prepared at $T_{\text {sub }}=0.8 T_{\mathrm{g}}$ and with a lower deposition rate than ref. ${ }^{9}$ (the star in Fig. 4). This value also collapses into the surface relaxation curve. Upon further lowering of the deposition rate, higher stability may most likely be obtained, leading to a lower value and thus an even better agreement.

Figure 4 provides a simple but ample look of the surface relaxation dynamics in glass formers, which is still relevant to

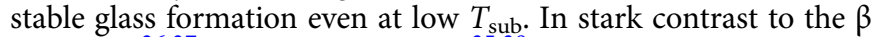
relaxation $^{36,37}$ and the a relaxation ${ }^{35,38}$ occurring in the bulk, the temperature dependence of surface relaxation is almost negligible, analogous to a recently discovered fast mode likely associated to local stress dipoles ${ }^{35}$. Although the nature of the surface relaxation cannot be elucidated within this study, our results suggest that even at low substrate temperatures, atomic motions at free surface remain still active and are many orders of magnitude faster than the structural rearrangements in the bulk, as shown in Fig. 4. Interestingly, previous measurements have shown that the surface relaxation time of glassy polymers exhibits no discernible temperature dependence when going somewhat below $T_{\mathrm{g}}$ (ref. ${ }^{13}$ ). Recently, it was found in ultrathin films of a molecular glass that the activation energy of the average film dynamics decreases dramatically with decreasing film thickness and eventually becomes even much smaller than that of the surface diffusion, suggesting as well the very weak temperature dependence of 
surface relaxation ${ }^{18}$. These findings agree with our suggestion that the dynamics at the glass surface is much faster than expected, even at low temperatures. A confirmation of this hypothesis will however require direct measurements of free surface relaxation dynamics in several glass formers. As multicomponent systems, it is likely that each element in MGs moves with a different timescale $^{39}$, even if STM measurements ${ }^{32-34}$ suggest more cooperative rearrangements at the surface. Further investigations are necessary to clarify this issue, as the knowledge of the mobility of each constituent element is important to better understand the surface mobility and the ultrastability.

Finally, it is worth stressing that the successful preparation of MGs with much improved stability at room temperature is of technological significance. MGs are regarded as extraordinary materials possessing unique mechanical and functional properties ${ }^{40,41}$. The use of high $T_{\text {sub }}$ during deposition brings additional technical problems as most MGs can only be deposited on substrates with high temperature resistance like $\mathrm{NaCl}^{9}$ or $\mathrm{Si}^{10}$. Consequently, their applications will be severely restricted if the substrate cannot stand high temperatures. This would be the case, for instance, for wear and corrosion resistant coating ${ }^{42}$, or in their use as components of composite materials. Our work not only provides access to ultrastable MGs at $T_{\text {sub }}$ far below $T_{\mathrm{g}}$, but also shows the experimental route necessary for obtaining even better stability, which will enable additional possibilities for wider technological applications.

In summary, we show the production of ultrastable MGs with increased stability by keeping $T_{\text {sub }}$ at room temperature $\left(\approx 0.43 T_{\mathrm{g}}\right.$ for $\mathrm{Zr}$-based MG), questioning therefore the general empiric rule of thumb of keeping the substrate at $0.8 \sim 0.9 T_{\mathrm{g}}$ (refs. ${ }^{5-12,19,21}$ ). By lowering the deposition rate to $\approx 1 \mathrm{~nm} \mathrm{~min}^{-1}$, we have been able to produce glasses with improved stability against crystallization, and with $\sim 60 \mathrm{~K}$ enhanced $T_{\mathrm{g}}$, significantly larger than that in conventional quenched glasses and in previous measured ultrastable $\mathrm{MGs}^{9}, 10$. Intriguingly, the stability improves on lowering the deposition rate until a low critical limit of $\approx 1 \mathrm{~nm} \mathrm{~min}^{-1}$. An estimation of the timescale associated to the surface dynamics provides a characteristic time of $\approx 17 \mathrm{~s}$, in good agreement with the data measured with STM in other MG systems. Structural analyses show that the ultrastable glasses are characterized by a more homogenous structure, with less order at larger distances, more stability and different phase formations during crystallization. The formation of stable glasses at $T_{\text {sub }}$ far below $T_{\mathrm{g}}$ will not only boost the scientific research on ultrastable glasses and surface relaxation, but may also allow defining a route to improve coatings on materials and design better amorphous alloys.

\section{Methods}

Metallic glasses preparation. Alloys with a normal composition of $\mathrm{Zr}_{46} \mathrm{Cu}_{46} \mathrm{Al}_{8}$ (at.\%) were prepared by arc-melting pure metals in a Ti-getter high-purity argon atmosphere. MG ribbons with $20 \mu \mathrm{m}$ thick were produced by melt-spinning technic.

MG films prepared by IBAD. The $\mathrm{Zr}_{46} \mathrm{Cu}_{46} \mathrm{Al}_{8}$ deposition target with a size of $100 \times 100 \times 2 \mathrm{~mm}^{3}$ is prepared by copper-mold-casting. Such a large area ensures the full coverage of the ion beam. Preceding the deposition of the MG, one layer of aluminum with $\sim 100 \mathrm{~nm}$ thick was firstly deposited on a flat polycarbonate (PC) plate with $\sim 12 \mathrm{~cm}$ in diameter, and then the chamber is opened for about $5 \mathrm{~min}$ for this aluminum layer to be exposed to atmosphere to form an oxidized layer on the surface. The formation of an oxidized aluminum layer makes the following deposited MG film being more easily detached. To strike off the possibly existing oxide layer on the target surface, pre-sputtering was carried out for $200 \mathrm{~s}$, then a MG film with $\sim 2 \mu \mathrm{m}$ thick was deposited. By tuning the ion beam current (i.e., from 2-70 $\mathrm{mA}$ ) we can obtain different deposition rates. The beam energy is $750 \mathrm{eV}$. The base pressure of the chamber is better than $2 \times 10^{-4} \mathrm{~Pa}$, the depositing argon pressure is $2.4 \times 10^{-2} \mathrm{~Pa}$. The as-received films were detached from the PC substrate by dissolving the in-between aluminum layer into a $1 \mathrm{~mol} \mathrm{~L}^{-1} \mathrm{NaOH}$ solution, and then the impurities on the films were removed using deionized water.
Effect of the substrate materials and comparison between different deposition techniques are discussed in Supplementary Note 1.

Differential scanning calorimetry analysis. A Perkin-Elmer DSC 8000 was employed to examine the kinetic and thermodynamics of the samples at a heating rate of $20 \mathrm{~K} \mathrm{~min}^{-1}$ with $20 \mathrm{~mL} \mathrm{~min}^{-1}$ flowing pure argon gas to prevent possible surface oxidation. The mass of DSC samples is $15 \sim 20 \mathrm{mg}$ to achieve good data accuracy. For each sample, at least three measurements were performed for statistical calculation of characteristic temperatures, e.g., the onset glass transition temperature, $T_{\mathrm{g}}$, and crystallization temperature, $T_{\mathrm{x}}$.

Near $\mathbf{T}_{\mathbf{g}}$ annealing. The annealing was conducted in a muffle furnace with temperature stability of $\pm 1 \mathrm{~K}$. Ordinary MG ribbons and $0.80 \mathrm{~nm} \mathrm{~min}^{-1}$ deposited films were studied. The samples were sealed in quartz tubes with high-purity argon gas to prevent oxidation and heated to the expected annealing temperatures at a rate of $20 \mathrm{~K} \mathrm{~min}^{-1}$. Three annealing procedures are studied at $700 \mathrm{~K}$ for $50 \mathrm{~h}$, at $759 \mathrm{~K}$ for $10 \mathrm{~min}$, and at $873 \mathrm{~K}$ for $10 \mathrm{~min}$. To fully crystallize the amorphous samples and compare their crystalline phase formation, we heated the samples at $20 \mathrm{~K} \mathrm{~min}^{-1}$ to $973 \mathrm{~K}$ and then annealed at this temperature for $1 \mathrm{~h}$. The annealed samples were examined by laboratory XRD to characterize their structures.

X-ray diffraction. The measured glass samples were put onto a zero-background silicon wafer, and laboratory XRD patterns were collected with a Bruker D8 Advance with a $\mathrm{Cu} K_{\alpha}$ source in the Bragg-Brentano geometry. High-energy XRD measurements were carried out with an incoming beam energy of $79.5 \mathrm{keV}$ at the ID15A beamline at ESRF, Grenoble (F). At $79.5 \mathrm{keV}$, the calculated X-ray attenuation for the $2 \mu \mathrm{m}$ thick deposited glass film varies from $0.17 \%$ at $q=0 \AA^{-1}$ to $0.24 \%$ at $q_{\max }=30 \AA^{-1}$. The calculated value for the $20 \mu \mathrm{m}$ thick ribbon varies instead from $1.7 \%$ at $q=0 \AA^{-1}$ to $2.4 \%$ at $q_{\max }=30 \AA^{-1}$. The attenuation is therefore linearly proportional to the sample thickness, weakly dependent on $q$, very small, and consequently negligible. The diffracted signal is also linearly proportional to the sample thickness. For such reasons, it is not necessary to use samples of similar thickness.

The incident flux normalization is done with a diode placed in front of the sample. Diffraction patterns were collected in transmission geometry by using a Pilatus3 X CdTe 2M hybrid photon counting detector. The detector was offcentered with respect to the incident beam and located close to the sample to access up to $q \approx 30 \AA^{-1}$ and to be able to calculate with a good resolution the pair distribution function $G(r)$. The intensity profiles were corrected for the background contribution, polarization of the X-rays, and detector geometrical correction. The $G$ $(r)$ and $S(q)$ were calculated using routines from the Diffpy-CMI library ${ }^{43}$ with some local modifications for outlier rejection and treatment of background effects.

Transmission electron microscopy. Specimens for TEM characterization were carefully prepared by ion milling with $3 \mathrm{keV}$ Ar ions at the liquid nitrogen temperature. HRTEM and HAADF-STEM observations were conducted using a cold field emission TEM (JEM-ARM200F, JEOL) equipped with a spherical aberration (Cs) corrector for the probe-forming objective lens.

Data availability. The data that support the findings of this study are available from the corresponding author upon request.

Received: 16 November 2017 Accepted: 2 March 2018

Published online: 11 April 2018

\section{References}

1. Debenedetti, P. G. \& Stillinger, F. H. Supercooled liquids and the glass transition. Nature 410, 259-267 (2001).

2. Dyre, J. C. Colloquium: the glass transition and elastic models of glass-forming liquids. Rev. Mod. Phys. 78, 953-972 (2006).

3. Badrinarayanan, P., Zheng, W., Li, Q. \& Simon, S. L. The glass transition temperature versus the fictive temperature. J. Non Cryst. Solids 353, 2603-2612 (2007)

4. Liu, Z. Y. et al. Cooling rate effect on Young's modulus and hardness of a Zrbased metallic glass. J. Alloys Compd. 509, 3269-3273 (2011).

5. Swallen, S. F. et al. Organic glasses with exceptional thermodynamic and kinetic stability. Science 315, 353-356 (2007).

6. Kearns, K. L., Still, T., Fytas, G. \& Ediger, M. D. High-modulus organic glasses prepared by physical vapor deposition. Adv. Mater. 22, 39-42 (2010).

7. Kearns, K. L. et al. Hiking down the energy landscape: progress toward the Kauzmann temperature via vapor deposition. J. Phys. Chem. B 112, 4934-4942 (2008). 
8. Yu, H. B., Tylinski, M., Guiseppi-Elie, A., Ediger, M. D. \& Richert, R. Suppression of $\beta$ relaxation in vapor-deposited ultrastable glasses. Phys. Rev. Lett. 115, 185501 (2015).

9. Yu, H. B., Luo, Y. \& Samwer, K. Ultrastable metallic glass. Adv. Mater. 25, 5904-5908 (2013).

10. Aji, D. P. et al. Ultrastrong and ultrastable metallic glass. Preprint at https:// arxiv.org/pdf/1306.1575 (2013)

11. Singh, S., Ediger, M. D. \& de Pablo, J. J. Ultrastable glasses from in silico vapour deposition. Nat. Mater. 12, 139-144 (2013)

12. Kearns, K. L., Swallen, S. F., Ediger, M. D., Wu, T. \& Yu, L. Influence of substrate temperature on the stability of glasses prepared by vapor deposition. J. Chem. Phys. 127, 154702 (2007).

13. Fakhraai, Z. \& Forrest, J. A. Measuring the surface dynamics of glassy polymers. Science 319, 600-604 (2008).

14. Chai, Y. et al. A direct quantitative measure of surface mobility in a glassy polymer. Science 343, 994-999 (2014).

15. Zhu, L. et al. Surface self-diffusion of an organic glass. Phys. Rev. Lett. 106, 256103 (2011)

16. Cao, C. R., Lu, Y. M., Bai, H. Y. \& Wang, W. H. High surface mobility and fast surface enhanced crystallization of metallic glass. Appl. Phys. Lett. 107, 141606 (2015).

17. Böddeker, B. \& Teichler, H. Dynamics near free surfaces of molecular dynamics simulated $\mathrm{Ni}_{0.5} \mathrm{Zr}_{0.5}$ metallic glass films. Phys. Rev. E 59, 1948-1956 (1999).

18. Zhang, Y. \& Fakhraai, Z. Decoupling of surface diffusion and relaxation dynamics of molecular glasses. Proc. Natl Acad. Sci. USA 114, 4915-4919 (2017).

19. Guo, Y. et al. Ultrastable nanostructured polymer glasses. Nat. Mater. 11, 337-343 (2012).

20. Yu, P., Bai, H. Y., Tang, M. B. \& Wang, W. H. Excellent glass-forming ability in simple $\mathrm{Cu}_{50} \mathrm{Zr}_{50}$-based alloys. J. Non Cryst. Solids 351, 1328-1332 (2005).

21. Chua, Y. Z. et al. How much time is needed to form a kinetically stable glass? AC calorimetric study of vapor-deposited glasses of ethylcyclohexane. $J$. Chem. Phys. 142, 054506 (2015).

22. Ma, G. Z. et al. Effect of Ti substitution on glass-forming ability and mechanical properties of a brittle $\mathrm{Cu}-\mathrm{Zr}-\mathrm{Al}$ bulk metallic glass. Mater. Sci. Eng. A 563, 112-116 (2013).

23. Zhu, L. \& Yu, L. Generality of forming stable organic glasses by vapor deposition. Chem. Phys. Lett. 499, 62-65 (2010).

24. Dawson, K. J. et al. Physical vapor deposition as a route to hidden amorphous states. Proc. Natl Acad. Sci. USA 106, 15165-15170 (2009).

25. Dawson, K. J. et al. Anisotropic structure and transformation kinetics of vapor-deposited indomethacin glasses. J. Phys. Chem. B 115, 455-463 (2011).

26. Pérez-Castañeda, T. et al. Suppression of tunneling two-level systems in ultrastable glasses of indomethacin. Proc. Natl Acad. Sci. USA 111, 11275-11280 (2014)

27. Rodríguez-Tinoco, C. et al. Highly stable glasses of celecoxib: influence on thermo-kinetic properties, microstructure and response towards crystal growth. J. Non Cryst. Solids 407, 256-261 (2015).

28. Zhu, F. et al. Intrinsic correlation between $\beta$-relaxation and spatial heterogeneity in a metallic glass. Nat. Commun. 7, 11516 (2016).

29. Liu, T. et al. Birefringent stable glass with predominantly isotropic molecular orientation. Phys. Rev. Lett. 119, 095502 (2017).

30. Tanaka, H. Relation between thermodynamics and kinetics of glass-forming liquids. Phys. Rev. Lett. 90, 055701 (2003).

31. Reid, D. R., Lyubimov, I., Ediger, M. D. \& de Pablo, J. J. Age and structure of a model vapour-deposited glass. Nat. Commun. 7, 13062 (2016).

32. Nguyen, D. et al. Composition-dependent metallic glass alloys correlate atomic mobility with collective glass surface dynamics. Phys. Chem. Chem. Phys. 18, 16856-16861 (2016).

33. Ashtekar, S., Scott, G., Lyding, J. \& Gruebele, M. Direct visualization of twostate dynamics on metallic glass surfaces well below $T_{\mathrm{g}}$. J. Phys. Chem. Lett. 1, 1941-1945 (2010).

34. Ashtekar, S., Lyding, J. \& Gruebele, M. Temperature-dependent two-state dynamics of individual cooperatively rearranging regions on a glass surface. Phys. Rev. Lett. 109, 166103 (2012).

35. Luo, P., Wen, P., Bai, H. Y., Ruta, B. \& Wang, W. H. Relaxation decoupling in metallic glasses at low temperatures. Phys. Rev. Lett. 118, 225901 (2017).
36. Luo, P. et al. Prominent b-relaxations in yttrium based metallic glasses. Appl. Phys. Lett. 106, 031907 (2015)

37. Yu, H. B., Wang, W. H., Bai, H. Y. \& Samwer, K. The $\beta$-relaxation in metallic glasses. Natl Sci. Rev. 1, 429-461 (2014).

38. Luo, P. et al. Probing the evolution of slow flow dynamics in metallic glasses. Phys. Rev. B 93, 104204 (2016).

39. Bartsch, A., Rätzke, K., Meyer, A. \& Faupel, F. Dynamic arrest in multicomponent glass-forming alloys. Phys. Rev. Lett. 104, 195901 (2010).

40. Ashby, M. F. \& Greer, A. L. Metallic glasses as structural materials. Scr. Mater 54, 321-326 (2006).

41. Wang, W. H. Bulk metallic glasses with functional physical properties. Adv. Mater. 21, 4524-4544 (2009).

42. Chu, J. P. et al. Thin film metallic glasses: unique properties and potential applications. Thin Solid Films 520, 5097-5122 (2012).

43. Juhás, P. et al. Complex modeling: a strategy and software program for combining multiple information sources to solve ill posed structure and nanostructure inverse problems. Acta Crystallogr. A 71, 562-568 (2015).

\section{Acknowledgements}

We are indebted to M. Gruebele and H. A. Nguyen for providing the STM data and helpful discussions. We gratefully thank M. Ediger, L. Yu, M. Gonzalez Silveira, and J. Rodriguez Viejo for stimulating discussions. Experimental assistance from Y. T. Sun, L. Q. Shen, Z. J. Wang, Y. C. Wu, and M. Liu are appreciated. ESRF is thanked for providing the beamtime. This research was supported by the NSF of China (11790291, 51571209, and 51461165101); the MOST 973 Program (2015CB856800), National Key Research and Development Plan (Grant No. 2016YFB0300501 \& 2017YFB0903902); and the Key Research Program of Frontier Sciences (QYZDY-SSW-JSC017) and the Strategic Priority Research of the Chinese Academy of Sciences (XDPB0601).

\section{Author contributions}

P.L. performed experiments on IBAD, DSC, laboratory XRD, discussed and analyzed the data and wrote the manuscript. C.R.C and Y.M.L. assisted in IBAD. F.Z. performed the TEM measurements. G.V. and M.D.M. carried out the synchrotron XRD measurements and the corresponding data treatment. B.R. discussed the data and wrote the manuscript. W.H.W. coordinated the project, analyzed the data, and wrote the manuscript. All authors contributed to comment on the manuscript writing and the result discussions.

\section{Additional information}

Supplementary Information accompanies this paper at https://doi.org/10.1038/s41467 018-03656-4.

Competing interests: The authors declare no competing interests.

Reprints and permission information is available online at http://npg.nature.com/ reprintsandpermissions/

Publisher's note: Springer Nature remains neutral with regard to jurisdictional claims in published maps and institutional affiliations.

Open Access This article is licensed under a Creative Commons Attribution 4.0 International License, which permits use, sharing, adaptation, distribution and reproduction in any medium or format, as long as you give appropriate credit to the original author(s) and the source, provide a link to the Creative Commons license, and indicate if changes were made. The images or other third party material in this article are included in the article's Creative Commons license, unless indicated otherwise in a credit line to the material. If material is not included in the article's Creative Commons license and your intended use is not permitted by statutory regulation or exceeds the permitted use, you will need to obtain permission directly from the copyright holder. To view a copy of this license, visit http://creativecommons.org/ licenses/by/4.0/.

(C) The Author(s) 2018 